\title{
EL COMPROMISO VALORATIVO DE LA TECNOLOGÍA
}

Luis A. Piscoya Hermoza

Universidad de San Marcos de Lima

Este artículo pretende ser una reflexión filosófica sobre la tecnología entendida en términos contemporáneos, vale decir, dentro del contexto científico que contiene, al menos, teorías, metodologías, sistemas lingüísticos, instrumentos de laboratorio y de campo. Asimismo, concebimos, en general, la tecnología como una variedad del conocimiento científico que tiene sentido operante o transformador, y que se expresa por medio de formulaciones prescriptivas conocidas como reglas tecnológicas.

Nuestro análisis se apoyará básicamente en argumentos lógicos, aunque no necesariamente en formalizaciones. Intentamos probar de este modo algunas tesis que difieren significativamente de puntos de vista cuasi tradicionales que tienen semejanza con algunos otros menos convencionales; en los resultados pero no en los mecanismos de prueba. En este segundo caso, nuestro aporte mínimo será reemplazar la aproximación intuitiva y difusa por el argumento explícito y riguroso.

Consideramos posiciones cuasi tradicionales, entre otras, aquellas que conciben la tecnología como una expresión altamente compleja del homo faber; que consiste esencialmente en la producción de instrumentos, maquinaria o artefactos que pueden utilizarse casi simétricamente para el beneficio o para el perjuicio, incluso el exterminio, de la especie humana. Dentro de esta concepción, los ingenieros aparecen como pro- 
veedores de artefactos o de información, como evaluadores de situaciones y proyectos; y los líderes sociales, empresariales o políticos, como los éticamente responsables de las decisiones (policy makers). Desde el punto de vista lógico, esta tendencia está asociada a aquella que tipifica las reglas tecnológicas como prescripciones pragmáticas, cuyo nivel no es de exigencia sino de recomendación y, consecuentemente, no entraña ni presupone compromiso ético valorativo alguno. ${ }^{1}$

Otro punto de vista, no incompatible con el anterior, es el que sostiene que la capacidad de la tecnología para producir artefactos la convierte fundamentalmente en creadora de nuevas opciones que hacen realizable, a veces muy fácilmente, lo que antes era concebido como prácticamente imposible o, simplemente, ni siquiera imaginado. De esta manera el "heracliteano" cambio tecnológico va creando formas de vida cada vez más complejas y ricas, que no pueden regirse ya por los valores correspondientes a etapas anteriores que cada vez con mayor rapidez se tornan en obsoletos. Así, la tecnología contemporánea sería el motor del cambio de valores y la configuradora del futuro, aunque ella en sí misma no sea valorativa sino creadora de opciones. 2

La tercera posición concibe la tecnología como fruto de un estilo especial de pensamiento calculante, que incluye también a la ciencia y a cierto tipo de filosofía, tal vez mayoritaria. Este pensamiento calculante sería a su vez represivo o bien encubridor de la genuina naturaleza humana, o bien del ser. De este modo, la tecnología sería el instrumento más poderoso de es-

1 Este punto de vista puede ser ilustiado por el artículo de D.C. Phillips y Nancy Sanders, Problems in Rational and Responsible Use of Evaluations, Occasional Papers of the Stanford Evaluation Consortium, School of Education, Stanford University, 1977.

2 Un ejemplo de este punto de vista lo proporciona Emmanuel Mesthene, "How Technology Will Shape the Future", en Carl Mitcham y Robert Mackey (comps.), Philosophy and Technology, The Free Press, 1983, pp. 116-129. 
ta forma de pensamiento que, dada su cobertura y generalidad, puede ser considerado como ideología. Desde esta perspectiva, el compromiso de la tecnología con los valores dominantes es total y necesario, como lo es también el de la ciencia y el de la filosofía de cepa positivista y sus variantes. Sin embargo, por su inmensa capacidad operante, la tecnología, desde este punto de vista, se convierte en la más seria amenaza a los genuinos valores humanos. ${ }^{3}$

Por nuestra parte, asumiendo explícitamente que la tecnología puede ser adecuadamente examinada a través de las reglas tecnológicas que la expresan, sostendremos como tesis de este trabajo las siguientes:

1. El sentido prescriptivo de las reglas tecnológicas y los predicados eficiente-ineficiente que usualmente se les atribuye, tienen como fundamento último un conjunto de criterios de valor que son expresión de lo que se considera socialmente bueno.

2. El contenido informativo de las reglas tecnológicas se apoya en enunciados científico-teóricos, en algún sentido verdaderos, pero ello no significa que los segundos impliquen lógicamente a las primeras, salvo que se admita explícitamente premisas adicionales de carácter valorativo y una implicación no clásica o no estándar.

3. La dependencia de las reglas tecnológicas de los sistemas de valores ha sido soslayada debido al énfasis puesto en el artefacto y no en el lenguaje. Las tecnologías conductuales han puesto en evidencia la capacidad operante notable de los sistemas simbólicos y su parcialidad valorativa.

La argumentación en pro de la plausibilidad de las tesis propuestas la desarrollaremos en tres partes. La primera estará

${ }^{3}$ Este punto de vista lo ilustran en medida significativa Martín Heidegger, Der Satz vom Grund, Friburgo, 1957; y Herbert Marcuse, El hombre unidimensional, Editorial Seix Barral, Barcelona, 1968. 
dedicada al análisis del lenguaje científico, que denominamos alético, para hacer los deslindes con el lenguaje no alético. Luego presentaremos el lenguaje de la tecnología, en términos de conjuntos de reglas de acción de naturaleza no alética. En la tercera parte, abordaremos lo relacionado con el compromiso valorativo de la tecnología como una consecuencia inherente a la naturaleza lógica de sus reglas.

\section{El lenguaje científico alético}

A pesar de la cautela que Reichenbach, Carnap y Tarski, entre otros, nos enseñaron a guardar frente al concepto de verdad, consideramos que se puede asumir que un vasto sector del lenguaje científico está constituido por proposiciones o enunciados que en algún sentido son susceptibles de ser verdaderos o falsos. No necesitamos discriminar, en este caso, peculiaridades de los llamados enunciados teóricos, o de los de observación, ni discutir lo que el concepto de verdad pueda significar en términos de grado de confirmación o de probabilidad, ni tomar posición respecto del mayor o menor grado de provisionalidad de la verdad de los enunciados. Nos basta con señalar que las proposiciones "la proposición $p$ es verdadera" y "la proposición $p$ es falsa" son significativas dentro de numerosos contextos que constituyen el lenguaje científico y todos ellos, por satisfacer esta condición, los denominamos aléticos, o decimos que gozan de la propiedad de aleticidad.

Asimismo, dentro del nivel alético, definiremos una teoría científica como un conjunto $T$ de proposiciones que satisface las siguientes condiciones: i) $T$ contiene sólo enunciados generales calificables de verdaderos; ii) $T$ contiene un conjunto mínimo de enunciados que son verdaderos, pero no son lógicamente deducibles dentro de $T$; iii) no se conocen enunciados singulares interpretables como contraejemplos de algún enunciado de $T$; y iv) para al menos un subconjunto no vacío de enunciados de $T$, se conoce un conjunto no vacío de enun- 
ciados singulares interpretables como ejemplos. Como se comprende, la generalidad de la definición anterior permite su uso sin mayores restricciones en las ciencias llamadas fácticas o en la lógica y en la matemática.

Decimos que una teoría $T$ es predictiva, si con ayuda de sus enunciados y a partir de descripciones de estados de cosas interpretables como estados iniciales, se puede predecir o descartar la ocurrencia de estados de cosas interpretables como finales. Una teoría $T$ es retrodictiva, si con ayuda de sus enunciados y a partir de descripciones de estados de cosas interpretables como estados finales, es posible la reconstrucción de presuntos estados iniciales. La aceptabilidad de una teoría científica con contenido empírico no exige el cumplimiento de las dos características anteriores. Así, por ejemplo, la teoría de la evolución en la biología y la teoría tectónica en la geología, que suscitaron dificultades epistemológicas cuando se intentó aplicarles la condición positivista de confirmación predicativa y la teoría popperiana de falsabilidad, que también es predicativa, no tendrían dificultades de aceptabilidad epistemológica en la medida en que puede bastarles ser retrodictivas.

Una teoría $T$ hace inteligible un sector de la realidad si es predictiva o si es retrodictiva en la medida en que las deducciones lógicas correspondientes son interpretables como explicaciones. Sin embargo, no consideramos que estos requisitos sean aditivos, en el sentido de que es posible contar con teorías pequeñas que sean al mismo tiempo predictivas y retrodictivas y con teorías de riqueza significativa, como la de la evolución, que son fundamentalmente retrodictivas, pero que han iluminado revolucionariamente nuestra comprensión de la realidad.

En la medida en que la tecnología se interesa esencialmente por la acción eficiente, las teorías relevantes para ella son las que ofrecen información precisa para saber a qué atenernos en una situación determinada. A la tecnología le interesa más la manejabilidad de las circunstancias que la comprensión de la realidad. Por ello, las teorías que son predictivas y 
retrodictivas, aunque pobres en contenido informativo, son más aprovechables por la tecnología que las teorías que hacen inteligibles vastos sectores de la realidad pero que sólo pueden ser difusamente retrodictivas. Esto último puede justificar la aceptabilidad del psicoanálisis, a pesar de opiniones en contra tan respetadas como las de Popper y Bunge, ${ }^{4}$ y explicar por qué existe una exitosa, aunque limitada, tecnología conductista. Exitosa porque posee capacidad predictiva estadísticamente significativa, y limitada porque se interesa metodológicamente sólo en la relación de covariación entre propiedades y no en la intelección o en la comprensión de las relaciones internas que pueden existir entre ellas. Lo anterior no presupone necesariamente que las teorías de contenido limitado pero preciso deban ser rivales de las teorías de mayor riqueza pero de menor exactitud, pues es posible obtenerlas como particularizaciones de estas últimas; es el caso de la hidráulica con respecto a la hidrodinámica, como lo ha señalado $\mathrm{M}$. Bunge. ${ }^{5}$

Consideramos que a los enunciados de las teorías científicas que satisfacen la caracterización anterior no les son aplicables, en sentido estricto, los predicados valorativos bueno-malo. De este modo, expresiones frecuentes aun dentro del vocabulario de algunos filósofos de la ciencia como "teoría buena" o "teoría mala", pueden ser traducidas por "teoría presuntamente verdadera" o "teoría verdadera" y por "teoría presuntamente falsa" o "teoría falsa". Esta traducción es propiamente una reducción que elimina la carga subjetiva inherente a los predicados valorativos que convierte éstos en superfluos dentro del contexto científico teórico.

4 La calificación del psicoanálisis de seudociencia se encuentra en Mario Bunge, La investigación científica, Ariel, Barcelona (Colección Convivium), 1972, vid. 1.6. Antes ya lo había hecho profusamente Karl Popper en La lógica de la investigación cientffica, Tecnos, Madrid, 1962. Se ratifica en Unended Quest, Open Court, Publishing Company, 1976.

5 Mario Bunge, op. cit., p. 686. 
El uso estricto de 'bueno' es inseparable de cierta exigencia de actitudes favorables, en el sentido de "tomar partido en favor de" o de "asumir una actitud o una posición en pro de". Para que nuestro punto de vista sea válido, no es necesario que la exigencia anterior agote el sentido de 'bueno' sino sólo admitir que es un componente estable de dicho predicado cuando se usa en afirmaciones relevantes. Así, por ejemplo, la afirmación "la democracia es buena" puede ser traducida, sin sufrir desnaturalización, por "se debe tomar posición en pro de la democracia". Y esta traducción puede ser entendida en términos de que es sostenible la doble implicación: "Si la democracia es buena entonces se debe tomar posición en pro de la democracia, y si se debe tomar posición en pro de la democracia entonces la democracia es buena". Asumiendo la pertinencia de la traducción anterior, que desde Frege ${ }^{6}$ es una equivalencia de significado más que de sentido, sería un despropósito traducir la oración "la teoría de la relatividad es buena" por "se debe tomar posición en pro de la teoría de la relatividad". Esto nos lleva a eliminar el uso valorativo de 'bueno' en el nivel alético y a sostener que cualquier aparición coloquial de dicho predicado que califica enunciados o teorías es interpretable adecuadamente en términos de 'verdadero'.

La delimitación anterior puede justificarse, como tradicionalmente se ha hecho, puntualizando que las teorías son formuladas con la pretensión de decir "lo que realmente ocurre" o simplemente "lo que es"; en este empeño nos proporcionan una imagen de la realidad, aunque ellas mismas en cuanto tales no sean imágenes de la realidad, sino sistemas o aparatos simbólicos para hacerla inteligible. Consecuentemente, las teorías no abren discusión sobre "lo que debemos favorecer" o sobre "lo que preferimos que sean", pues su sentido es mostrativo y no prescriptivo.

6 G. Frege, Funktion, Begriff und Bedeutung, Vandenhoeck und Reuprecht, Gotinga, 5a. ed., 1980, pp. 40-65. 
En relación con expresiones que hablan de los objetivos malos o buenos de las investigaciones que dieron lugar a la formulación de una teoría, o de los medios buenos o vedados utilizados en la realización de experimentos para contrastar hipótesis, en estos casos hablamos de las intenciones buenas o malas del investigador o de su conducta moral o inmoral, pero no del valor alético de los enunciados de la teoría que es independiente de las condiciones subjetivas, o de las peripecias conductuales del investigador. La responsabilidad social y moral es del investigador, no de la teoría.

\section{El nivel de la eficiencia}

Dentro del lenguaje científico, tomado en el sentido amplio, es posible distinguir el lenguaje de la tecnología, cuya característica relevante es ser prescriptivo y no mostrativo. A la tecnología le interesa fundamentalmente solucionar eficientemente problemas prácticos que se originan en las necesidades sociales. La satisfacción de estas necesidades constituye la meta que da sentido y dirección a la prescripción tecnológica que recomienda un curso de acción por el beneficio que produce. Por ello no le interesa la comprensión de la realidad, sino la manipulación o transformación de la misma para el logro de lo deseado.

El lenguaje de la tecnología está constituido por reglas de acción que según una propuesta de Mario Bunge tienen la estructura $B$ por $A$, que según él mismo se lee $B$ por medio de $A$ o también "para obtener $B$, hacer $A$ ". Este esquema recoge el sentido operante o instrumental de la regla tecnológica, pero es insuficiente para dar cuenta de su sentido normativo o prescriptivo que, ciertamente, Bunge no ignora sino que explícitamente reconoce. En efecto, con relación a las que él denominaba sin reservas teorías tecnológicas, afirma: "they are

7 Mario Bunge, op. cit., p. 697. 
concerned with finding out what ought to be done in order to bring about, prevent or just change the pace of events or their course in a preassigned way". ${ }^{8}$ Evidentemente, el sentido prescriptivo de "ought to" no deja lugar a dudas y es compatible totalmente con el punto de vista que se sostiene en el trabajo citado. Sin embargo, nos permitimos anotar que en la correspondiente versión española de Manuel Sacristán se usa la expresion "hay que", que debilita el sentido normativo de "ought to". 9 Asimismo, nosotros en este trabajo y en anteriores hemos preferido no usar el término 'teoría tecnológica' por no parecernos compatible con el carácter eminentemente prescriptivo de la tecnología. En un trabajo posterior, Bunge ha preferido omitir tal término y sugerir la pertinencia de su uso como una cuestión problemática. ${ }^{10}$

Un análisis adecuado de la regla tecnológica debe realizarse a partir de un esquema lógico que recoja su especificidad prescriptiva. No es suficiente puntualizar que se logra $B$ por medio de $A$, pues en la práctica pueden existir $A_{1}, A_{2}, \ldots, A_{n}$ para obtener $B$. Por ello, la regla tecnológica debe descartar los $A_{i}(1 \leq i \leq n)$ que son lentos, laboriosos y costosos y prescribir concretamente un $A_{k}$ o una secuencia $A_{k_{1}}, A_{k_{2}}, \ldots, A_{k_{n}}$ para el logro de $B$.

Por otra parte, si bien la intención del logro de un objetivo explica adecuadamente el sentido prescriptivo de una regla tecnológica, no debería omitirse que los estados iniciales son importantes para comprender el contenido prescrito en situaciones particulares. Es claro que el logro de un mismo objetivo puede dar lugar a recomendaciones diferentes, si las condiciones de partida son también diferentes. Debido a estas consideraciones, nosotros hemos propuesto como esquema general de

8 Mario Bunge, "Toward a Philosophy of Technology", en Mitcham y Mackey (comps.), Philosophy and Technology, p. 63.

9 Idem, La investigación cientffica, p. 686.

10 Idem, Epistemologia, Ciencias Sociales, La Habana, 1982, p. 215. 
la regla tecnológica el siguiente: "en las circunstancias $X$ debe hacerse $Y$ para $Z$ ". ${ }^{11}$ De esta manera, resolvemos la cuestión planteada por el sentido prescriptivo de la regla tecnológica por medio de 'debe', que en este caso tiene un sentido eminentemente instrumental. Igualmente, pensamos que dado un contexto específico, es suficiente con una formulación simplificada o elíptica del esquema anterior que omita la referencia a circunstancias. En tal caso bastará el esquema "debe hacerse $Y$ para $Z$ ".

Mantener explícito el carácter prescriptivo de la regla tecnológica es decisivo para establecer diferencias sustantivas con los enunciados que constituyen las teorfas científicas. Así, por ejemplo, carece de sentido decir que una recomendación o prescripción para la acción sea susceptible de ser verdadera o falsa, pues ella sólo puede ser seguida, cumplida, ignorada o transgredida por seres humanos. La regla tecnológica no se refiere a hechos en general, sino prescribe acciones o formas de conducta para el logro de objetivos. Si la acción prescrita conduce al logro del objetivo propuesto, entonces la regla correspondiente es eficiente, en caso contrario la regla es ineficiente. La no aleticidad de las reglas consiste justamente en que ellas, debido a su sentido prescriptivo, no pueden ser calificadas de verdaderas o falsas sino de eficientes o ineficientes, lo que pone de relieve su carácter utilitario o pragmático. Al mismo tiempo, la dicotomía eficiente-ineficiente deja abierta la posibilidad, interesante desde el ángulo de las necesidades prácticas, de definir grados de eficiencia en términos métricos. Ciertamente, en la práctica concreta la elección más frecuente parece ser no entre reglas eficientes e ineficientes, sino entre más eficientes y menos eficientes. Una teoría sobre los grados de eficiencia, sistematizada en términos del cálculo de probabilidades, tropezaría con menos dificultades que una teoría so-

11 Luis Piscoya, Sobre la naturaleza de la pedagogí, Retablo de Papel Ediciones, INIDE, Lima, 1974, p. 109. 
bre los grados de verdad, que es lo que finalmente trató de hacer Reichenbach en su discutido y voluminoso trabajo The Theory of Probability. ${ }^{12}$

El grado de eficiencia de las reglas tecnológicas está fuertemente ligado al conocimiento científico-térico disponible. No parece gratuito que las tecnologías más eficientes, como la electrónica, estén ligadas a los sectores de la ciencia teórica que ofrecen altos niveles de precisión y que tratan problemas que admiten soluciones mediante algoritmos. En concreto, es el caso de la tecnología de diseño de circuitos lógicos para computadoras, que aprovecha el álgebra de Boole. Sin embargo, una teoría altamente precisa y aplicable exitosamente a la tecnología no significa necesariamente una teoría cognoscitivamente rica. Esto acontece justamente con el álgebra de Boole, que es una estructura pequeña dentro de la lógica matemática pero tecnológicamente muy rentable.

El grado de eficiencia de las reglas tecnológicas, por consiguiente, descansa significativamente en el grado de exactitud y precisión con que están formuladas las proposiciones teóricas que le sirven de apoyo. Esto significa que, para la tecnología, más interesante que la verdad misma de las proposiciones teóricas, es el lenguaje en el que están formuladas tales proposiciones verdaderas. Esto hace comprensible que las tecnologías más eficientes sean las que están conectadas con teorías expresadas en lenguaje matemático. Sin embargo, no pretendemos que el grado de eficiencia de una regla tecnológica se derive lógicamente del grado de precisión de ciertas proposiciones teóricas, ni menos de su verdad. Ello se debe a que parece artificioso separar la eficiencia del objeto de la regla y hacerla depender exclusivamente de la calidad de la información científico-teórica. En efecto, la conexión entre grado de eficiencia y objetivo es clara, si se considera que la información

12 Hans Reichenbach, The Theory of Probability, University of California Press, 1949. 
científica útil para el logro de un determinado objetivo puede ser menos útil o menos relevante si modificamos el objetivo o lo sustituimos. Este nexo muestra que manteniendo constante la información científica que porta la regla, expresada por medio de la acción que prescribe, modificamos significativamente su grado de eficiencia si modificamos el objetivo de la misma. Podría argumentarse que si modificamos el objetivo de una regla, entonces ya estamos hablando en sentido estricto del grado de eficiencia de otra. Pero ello no modificaría la validez de nuestro planteamiento que podría tomar la siguiente forma: si, dadas al menos dos reglas tecnológicas que coinciden exactamente en todo excepto en el objetivo, ocurre que si presentan grados diversos de eficiencia, entonces debemos pensar que estas variaciones están relacionadas con la variación sufrida por el objetivo, lo que significa que el grado de eficiencia de las reglas tecnológicas no puede ser explicado suficientemente por el grado de exactitud y precisión de las proposiciones científico-teóricas en las que dichas reglas se apoyan. Éste es, a nuestro juicio, un argumento favorable a nuestra tesis 2 .

Lo antes sostenido no es incompatible con que el grado de eficiencia de una regla tecnológica pueda usarse como elemento de juicio en favor de la verdad de una determinada proposición científico-teórica.

\section{Prescripción tecnológica y valores}

En la sección anterior, hemos aportado argumentos probatorios de que el grado de eficiencia de la regla tecnológica está asociado a la precisión y exactitud de determinados enunciados científico-tecnológicos y a la naturaleza del objetivo que da sentido teleológico a la regla en referencia. En esta sección, intentaremos probar centralmente nuestra tesis 1 sosteniendo, por un lado, que lo que explica el nexo entre los grados de eficiencia y la naturaleza del objetivo es el sentido prescriptivo de la regla y, por otro lado, que el sentido prescriptivo de la re- 
gla es independiente en medida significativa de la información teórica y dependiente principalmente del objetivo que se desea alcanzar.

Iniciaremos nuestro análisis con un ejemplo. Una formulación aceptable de una regla tecnológica puede ser la siguiente: "En circunstancias en las que estemos ante pacientes no especialmente alérgicos, debe aplicárseles estreptomicina para curar la tuberculosis". Podemos suponer que esta prescripción se funda en que la investigación médico-farmacológica ha establecido que hay una especie de ajuste entre el medio y el objetivo o fin. Esta relación de ajuste instrumental se funda, en este caso, en una relación causal: en el sentido de que la estreptomicina produce un proceso de destrucción de los bacilos de Koch. Sin embargo, la conexión causal no implica prescripción alguna, pues ella nos informa que la estreptomicina destruye los bacilos de Koch, pero esto no implica que debamos tener interés en curar a los enfermos de tuberculosis. Es más, poniéndonos en una hipotética situación maligna, no necesariamente extraña a nuestra experiencia histórica, podríamos utilizar esta información para impedir que seres enfermos de tuberculosis se curen si lo que nos importa es exterminarlos. Consecuentemente, prescribimos estreptomicina bajo las circunstancias descritas, porque deseamos curar la tuberculosis, esto es, nos interesa la supervivencia de los afectados. $\mathrm{Y}$ es en la medida en que asumimos tal objetivo como válido que tiene sentido decir "debe". Por tanto, la investigación bioquímica explica la conexión causal y el ajuste entre medios y fines, pero no el "debe" que es el elemento que da sentido prescriptivo a la regla. Y este sentido prescriptivo nos permite hablar de la eficiencia, en tanto que ella expresa la capacidad misma para lograr un objetivo y pierde sentido si se independiza de él.

$\mathrm{El}$ análisis del ejemplo anterior explica suficientemente que en tal regla el uso de "debe" está justificado por el objetivo o fin. Podría contraargumentarse diciendo que nuestras conclusiones no poseen generalidad significativa debido a que hemos 
escogido un ejemplo ad hoc que le otorga un especial nivel de exigencia al "debe", por tratarse de un fin que más que práctico es moral. En cambio, si se recurriera a un ejemplo como el que presenta Mario Bunge en el trabajo antes citado, la situación es muy distinta. ${ }^{13}$ Utilizando nuestro esquema el ejemplo referido puede reformarse así: "En condiciones de un aceptable control experimental, debe mantenerse un cuerpo por debajo de su punto de Curie para mantenerlo imantado". En este caso se trataría de una regla tecnológica presuntamente más representativa que la propuesta por nosotros, porque el objetivo de mantener un cuerpo imantado es totalmente pragmático y carece de resonancia moral alguna.

Sin embargo, esa contraargumentación sería inexacta. El fin puede ser entendido en términos puramente utilitarios, pragmáticos, inmediatistas o de otro modo. Para nuestro punto de vista, es suficiente que se admita, como lo hacen aun los pragmatistas radicales, que el fin es deseado sin importar por ahora las razones. La deseabilidad del fin, nos obliga a expresarnos en términos de prescripciones o recomendaciones. De no ser así, nos bastarían los enunciados; pero está suficientemente admitido que con ellos describimos y no prescribimos. Consideramos que desconocer esta delimitación no resolvería problema alguno y crearía grandes confusiones.

La deseabilidad del fin puede entenderse como algo fundado en su bondad. Esto es, todo fin u objetivo de la acción humana es deseado en tanto se considera bueno en algún sentido. Resultaría una incoherencia en términos de la razón práctica afirmar " $X$ es malo y yo deseo $X$ ", pues la deseabilidad de $X$ es indicadora de que en algún otro respecto no hecho explícito se considera bueno. Por consiguiente, es la bondad del fin el fundamento del sentido prescriptivo de las reglas tecnológicas, aunque ello no implica que tal bondad sea siempre de la misma naturaleza ni que todas las reglas obliguen de la misma mane-

Mario Bunge, La investigación cientifica, p. 696. 
ra. Por otra parte, no es válido identificar todas las formas de "debe" con un sentido moral, pues aunque la afirmación "toda forma de "debe' moral es una forma de 'debe' ligada a valores" es válida, la inversa "toda forma de "debe' ligada a valores es una forma de 'debe' moral" no está implicada por la primera.

$\mathrm{El}$ análisis anterior pone en evidencia que las relaciones entre tecnología y criterios de bueno son insoslayables, debido a que tal vínculo se desprende de la naturaleza misma de las reglas tecnológicas. El hecho de que tal temática no haya recibido el tratamiento que merece puede deberse a que las tendencias tradicionales ven la tecnología a través de los artefactos que produce y no a través de los sistemas de reglas que hacen posible la construcción de tales artefactos. Asimismo, las cuestiones relacionadas con la transferencia de tecnología son con frecuencia discutidas desde el ángulo de la transferencia de maquinarias o de la concesión de patentes de fabricación. De este modo, quedan encubiertas propiedades que resultan de reconocimiento ineludible cuando el analista se dirige hacia el lenguaje de la tecnología.

El papel decisivo que cumplen nuestros criterios de bueno en nuestra concepción de la tecnología es detectable si nos remitimos a la experiencia social. Así, puede explicarse por qué no reconocemos a Josef Mengele como un tecnólogo sino como un genocida, mientras para el partido Nacional Socialista alemán era un científico muy respetable por su capacidad para construir eficientes máquinas de exterminio. Lo que explica tan importante diferencia es que nuestros criterios de bueno difieren sustancialmente de los dominantes en el Tercer Reich. Y la razón por la que los trabajos de Mengele se realizaron es porque existió un sistema de valores que permitió calificarlos de tecnología. De este modo, la conexión de los objetivos de las reglas tecnológicas con los criterios de bueno no es casual, en la medida en que ellos funcionan como condición de posibilidad para el desarrollo de la tecnología. Y si bien es cierto que esta ligazón es especialmente visible en casos extremos co- 
mo el mencionado, ello no impide que esté subyacente en las tecnologías posibilitadas por los criterios de bueno comunes a sistemas de valores diversos. Esto significa que cuanto más diferentes son los sistemas de valores de dos comunidades, tanto más difícil es la transferencia de tecnología entre ellas.

Naturalmente, la validez de nuestro aserto, que hemos denominado tesis 1 , no presupone que los tecnólogos sean conscientes de este condicionamiento. Muchos de ellos se sentirán "más allá de la libertad y de la dignidad" o "más allá del bien y del mal". 14 Empero, no tener conciencia de la ocurrencia de un proceso no suprime su existencia ni los riesgos que puede conllevar.

A fin de proporcionar una prueba completa de nuestra tesis 1 , a continuación trataremos de delinear la reconstrucción racional de la inferencia tecnológica o de la deducción tecnológica en un sentido muy próximo al que propuso Reichenbach $^{15}$ hace ya más de 50 años. Nos proponemos construir la deducción que hace posible la obtención de reglas tecnológicas, aunque ella no se inscriba dentro de la lógica clásica. Esto no quiere decir que los tecnólogos propongan reglas por medio de un proceso lógico explícito, sino que es posible construir una especie de artificio para hacer lógicamente inteligible su proceder.

Partiremos del hecho de que si la regla tecnológica tiene sentido prescriptivo, entonces nos encontramos ante una deducción con una conclusión formulada en términos de un "debe" que en alguna medida está condicionado valorativamente, aunque no necesariamente moralmente, como se desprende de nuestro análisis. Asimismo, la interpretación pragmatista, instrumental o utilitaria de "debe", como creemos haberlo demostrado, no es independiente del concepto de bueno. La presencia

14 Aludimos indirectamente a los libros publicados bajo tales títulos por B.F. Skinner y Federico Nietzsche.

$15 \mathrm{H}$. Reichenbach, Experience and Prediction, The University of Chicago Press, 1961, p. 8 y ss. La primera edición es de 1938. 
de una conclusión normativa nos trae reminiscencias de la vieja "guillotina de Hume", que advertía la imposibilidad de la referida conclusión desde premisas formuladas en términos de "es". La reformulación de esta prohibición establecería la imposibilidad de la conclusión referida desde premisas formuladas en términos de "es" y de "si..., entonces...", pues las hipótesis que forman parte de una teoría tienen forma lógica condicional y sólo los informes de observación pueden ser formulados mediante enunciados básicos en términos de "es". Esta actualización, sin embargo, no cambia la prohibición en lo fundamental. No parece tampoco justificable el paso de "si..., entonces..." a "debe".

La manera más simple de superar la dificultad sería establecer la mediación entre "si. .., entonces...", "es" y el "debe" de la conclusión. Ello sería mediante la adición de una premisa valorativa en términos de "debe" y de la aceptación explícita de que todo enunciado valorativo, relevante en términos de "bueno", tiene capacidad para justificar el logro o la realización de aquello considerado bueno.

De esta manera, una deducción tecnológica reconstruida sería una estructura constituida por: i) hipótesis científicas que portan información teórica relevante; ii) al menos un enunciado valorativo formulado en términos de "bueno"; iii) enunciados observacionales básicos o protocolarios; y iv) una norma de acción denominada regla tecnológica como conclusión obtenida a partir de i), ii) y iii). A las reglas de deducción usualmente aceptables habría que añadir que un enunciado valorativo en términos de "bueno" posibilita la deducción de una regla en términos de "debe". Ciertamente, el contenido específico de "bueno" se define dentro de un contexto que admite una jerarquización de lo valioso. Asimismo, no presuponemos en modo alguno la validez de los criterios de bueno que usa el tecnólogo, sino que señalamos que su razonamiento tiene entre sus fundamentos algún concepto de bueno. Por añadidura, tampoco estamos sugiriendo que la justificación lógica sea al 
mismo tiempo justificación moral, pero el tratamiento de este último tema excede los alcances de este trabajo.

Si nuestra reconstrucción del razonamiento tecnológico es correcta, contamos con un argumento fuerte para sostener nuestra tesis 1, que no sólo tendría el apoyo del análisis de la existencia social realizado antes. De este modo, estaríamos probando que en las bases racionales de la tecnología hay una decisión valorativa, consciente o no, que la compromete en sus fundamentos mismos con un determinado sistema de valores y que, en consecuencia, el viejo problema de la neutralidad valorativa de la tecnología puede estar solucionado negativamente. Abreviando, no es lógicamente posible una tecnología más allá del bien y del mal. 


\section{SUMMARY}

This paper is a critical analysis of technology as understood today. The author tries to prove three theses: 1) Prescriptive meaning of technological rules, and efficient-inefficient statements are based on expressing what is socially good. 2) Information contained in technological rules is based on scientific-theoretical statements-true in some sense. 3) Technological rules dependence upon systems of value has been avoided because it places emphasis on the artifact and forgets the language.

The three theses are based on the analysis of scientific language. They present technology language as action rules of non-scientific nature, and they approach the valorative commitment of technology as if it was an inherent consequence of the logical nature of its own rules.

[Carolina Celorio] 